\title{
A Novel High-Sensitivity Butterfly Gyroscope Driven by Horizontal Driving Force
}

\author{
Xu Qiang, Xiao Dingbang*, Hou Zhanqiang, Zhuo Ming, Li Wenyin, Xu Xiangming, Wu Xuezhong \\ College of Intelligence Science and Engineering, National University of Defense Technology \\ Faculty of Physical Sciences and Engineering, University of Southampton, UK \\ Changsha, Hunan, China \\ dingbangxiao@nudt.edu.cn
}

\begin{abstract}
Sensitivity is an important performance index of micro-machined gyroscope, and driving amplitude and initial capacitance gap are related to the signal-noise ratio as well as the sensitivity of gyroscope. The traditional butterfly gyroscope in our research group will generate both horizontal and vertical driving amplitude when driven in driving mode. So, the signal-noise ratio is subjected to the vertical driving displacement and initial capacitance gap, which, to a great extent, limits its sensitivity. In this paper, a novel high-sensitivity butterfly gyroscope with rectangle vibration beam was proposed, which will generate almost only horizontal displacement due to the structural characteristics when driven by horizontal driving force. Therefore, the proposed structure can not only increase the driving amplitude, but also reduce the initial capacitance gap, so as to enhance the signal-noise ratio as well as sensitivity of the gyroscope. After the structural design and manufacture processing, the performance of the proposed butterfly gyroscope was tested, and the angle random walk (ARW) is measured to be $1.244 \mathrm{deg} / \sqrt{\mathrm{h}}$, and the bias stability reached $3.58 \mathrm{deg} / \mathrm{h}$.
\end{abstract}

Keywords-butterfly micro-machined gyroscope, rectangle vibration beam, driving amplitude, signal-noise ratio

\section{INTRODUCTION}

At present, inertial gyroscopes mainly include mechanical rotor gyroscope, electrostatic gyroscope, laser gyroscope, fiber optic gyroscope (FOG), micro-machined gyroscope and other gyroscopes. The fiber optic gyroscope and laser gyroscope are one of the high-performance gyroscopes among them. But they're difficult to miniaturized the volume and produce in large quantities. The precision of mechanical rotor gyroscope and electrostatic gyroscope can reach the inertial level, but the application is limited to their high price and long startup time. Benefiting from the small size, low cost, low power consumption and batch fabrication, the micro-machined gyroscope has enjoyed great importance by all countries over the past 20 years. And the micro-machined gyroscope has been widely used in the fields of electronics consumer, vehicle safety, weaponry and other fields [1-3].

Over the past decades, a significant amount of research on the vibratory gyroscope with different kinds of structures has been directed towards the development of high performance and small size MEMS gyroscopes for various applications. The typical structures of MEMS gyroscopes include turning fork [48], comb [9-11], ring or disk [12-15], micro wineglass [16-18], etc. Among them, a structure called "Butterfly" gyroscope was firstly proposed by G. Andersson [19, 20]. Details of the fabrication process and basic working principles have already been reported in our previous works [21-23]. Without multisuspension beams and anchors, this structure uses only one slanted beam for suspension, which is simple to be manufactured. When the gyroscope is excited, vertical electrostatic forces bend the slanted beam both vertically and horizontally. When an angular rate is applied, the horizontal component of velocity vector produces vertical Coriolis force and vertical movement, which can be detected by the capacitive variety. With the help of the slanted beam, the gyroscope realizes single side electrostatic excitation and capacitive detection, which also reduces the complexity of fabrication process. Due to the advantages mentioned above, the gyroscope has attracted lots of interest ever since it is proposed. Many control methods are also present to improve the performance, including force feedback for the detection mode [24-26], dynamic electrical balancing of coupling stiffness [22], and effect of axial force [27], and so on.

For all that, the micro-machined gyroscope which can meet the requirements of inertial level is rarely reported. The sensitivity of the micro-machined gyroscope is one of the most important performance parameter. On the one hand, the range of use of gyroscope is affected by its sensitivity. Only those gyroscopes that can meet the requirements of high sensitivity can be used in the application field of small variation in angular velocity. On the other hand, the sensitivity is directly related to the overall noise level of the gyro. And those gyroscopes with high signal-noise ratio will result in high sensitivity, which can finally improve the performance of gyroscopes.

At present, the vibration beam of conventional butterfly micro-machined gyroscopes in our research group is a parallelogram beam which is fabricated by wet-etching. Driven by the vertical driving force, the vibration structure will generate both horizontal and vertical displacements caused by driving force. When the driving amplitude is large enough, the vertical displacement will be so large that the vibrating mass will collide with the glass plate, which directly limits the magnitude of the driving amplitude. Furthermore, the gap between vibration structure and glass (initial capacitive gap) is also limited to the vertical displacement generated by driving force.

In order to solve the problem and increase the driving amplitude, a novel vibration structure of micro-machined gyroscope was presented. The sectional view of the proposed vibration beam is a rectangle instead of the traditional one (a 
parallelogram), and the driving electrostatic force is changed from vertical direction to horizontal direction, which could increase the horizontal displacement and decrease the vertical displacement. Therefore, these following benefits can be brought:

Firstly, when driven in driving mode, the proposed vibration structure will generate almost only horizontal displacement and no obvious vertical displacement. So, the amplitude of driving vibration can be further increased to enhance the signal-noise ratio as well as sensitivity of gyroscope without worrying about the collision. Secondly, the initial capacitive gap can be further reduced without obvious vertical displacement, which can also increase the signal-noise ratio as well as the sensitivity of the gyroscope.

\section{DESIGN OF VIBRATION STRUCTURE}

The main idea of this proposed vibration structure is to enlarge the horizontal driving amplitude and reduce the vertical driving amplitude so as to enhance the signal-noise ratio as well as the sensitivity of the gyroscope by designing its vibration structure.

\subsection{The principle of the conventional butterfly gyroscope}

The conventional butterfly micro-machined gyroscope in our research group consists of a silicon structure with a parallelogram vibration beam and a glass substrate deposited with metal electrodes, shown in Fig. 1. The structure of the gyroscope is manufactured by wet etching from both sides in a single crystal silicon substrate, and the cross-section of the vibration beam is a parallelogram whose spindle azimuth $\left(\theta_{p}\right)$ is $54.74^{\circ}[21]$. When driven by vertical driving force generated between the mass and drive electrode, the structure would generate both horizontal and vertical displacements.

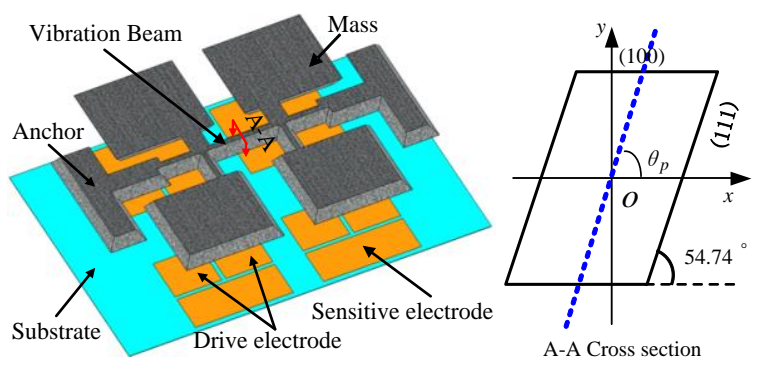

Fig. 1. Conventional butterfly gyroscope in our research group

The Coriolis effect is based on conservation of momentum. By applying a sinusoidal voltage with a direct current bias to the electrodes deposited on the glass substrate, a momentum is induced. The drive mode vibrates on its resonant frequency and achieves a stable amplitude and phase by the use of phase lock loop (PLL) and automatic gain control (AGC) methods. Inertial energy is transferred from drive mode to sense mode when the Coriolis effect is induced. Due to the Coriolis effect, there will be a sinusoidal signal in sense mode, which reflects the value of the input angular rate.

The modal simulation result of the conventional butterfly gyroscope is shown in Fig. 2.

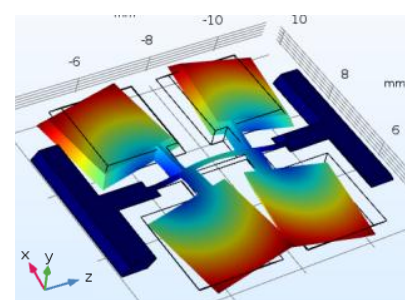

(a) Driving mode

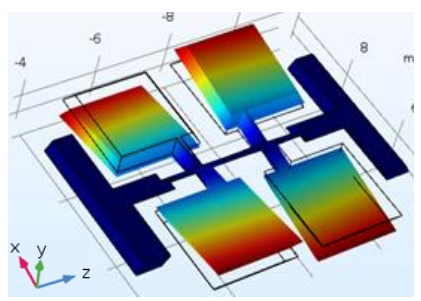

(b) Sensing mode
Fig. 2. Modal simulation result of the conventional butterfly gyroscope

As can be seen from the Fig. 2 (a), when the gyroscope is driven in driving mode, the vibrating mass will generate both horizontal and vertical displacement. However, due to the ingenious symmetrical design of driving electrodes, the mass will rotate around its center as shown in Fig. 3. So, the capacitance variation caused by the vertical driving force could not be detected by the sensing electrodes and will not introduce any quadrature.

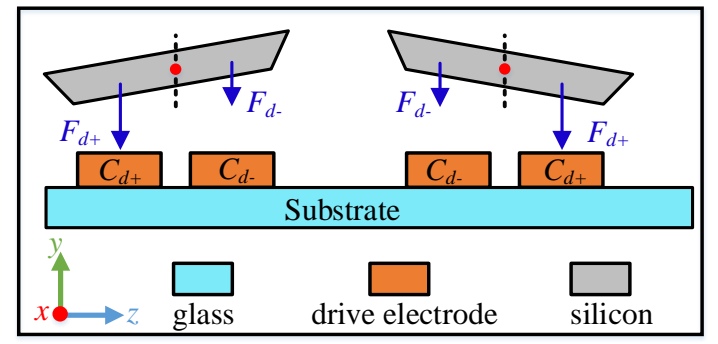

Fig. 3. The displacement of the mass and the relative positon of the electrodes in the drive mode

When excited in driving mode, the mass will generate both vertical and horizontal driving displacement components, as shown in Fig. 4. And the maximum vertical component (MaxdriveY) is $1 / 3$ of the initial capacitance gap (d), due to the pull-in effect. So, the maximum horizontal component (MaxdriveX) can be calculated according to the MaxdriveY $(d / 3)$ and spindle azimuth $\left(\theta_{p}\right)$ :

$$
\text { Maxdrive } X=k \cdot \frac{d}{3} \cdot \tan \left(\theta_{p}\right)
$$

The $k$ in the equation (1) is a coefficient that is only associated with the structural parameters of gyroscope. So we can see that, if the other parameters are constant, MaxdriveX would become larger, as the $\theta_{p}$ grows larger. And when the $\theta_{p}$ is up to $90^{\circ}$, the MaxdriveX would be extremely large in theory. Therefore, we could obtain more driving displacement and achieve a better signal-to-noise ratio of the gyroscope by a new design with bigger spindle azimuth angle $\theta_{p}$.

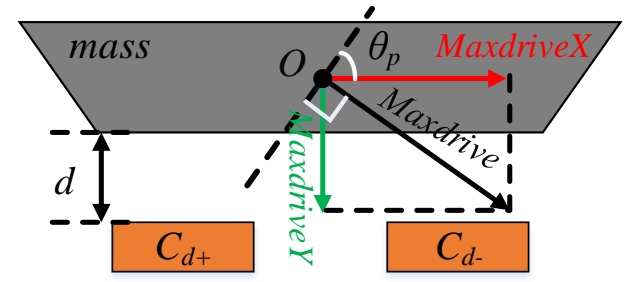

Fig. 4. Vertical and horizontal component of driving displacement 


\subsection{Overall design of the proposed gyroscope}

The proposed butterfly micro-machined gyroscope is fabricated by deep reactive ion etching (DRIE) processing, shown in Fig. 5. The sectional view of its vibration beam is a rectangle whose spindle azimuth was $90^{\circ}$. Due to the structural characteristics of the rectangle vibration beam, the proposed gyroscope should be driven by horizontal driving force instead of vertical driving force. So, if driven by horizontal driving force, the vibration structure would generate only horizontal displacement and no vertical displacement.

In order to achieve the horizontal driving force, a type of comb teeth structure is designed on the mass of the vibration structure and the corresponding electrodes are designed on the glass plate to generate the horizontal driving forces.

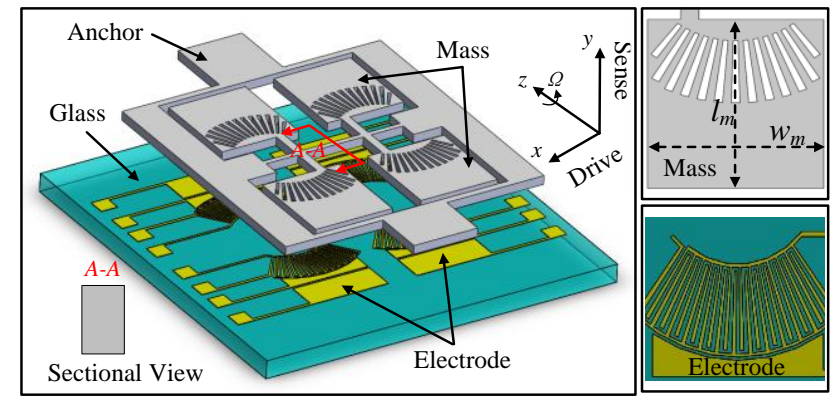

Fig. 5. The overall model of proposed vibration structure

When driving voltage is applied to the electrodes, electrostatic forces will be generated in two different directions between the comb teeth on silicon and electrodes on glass, as shown in Fig. 6. $F_{n}$ is the vertical electrostatic force, $F_{r}$ is the horizontal electrostatic force, $a$ is the overlap length, $b$ is the length of comb tooth, $c$ is the width of one single comb tooth.
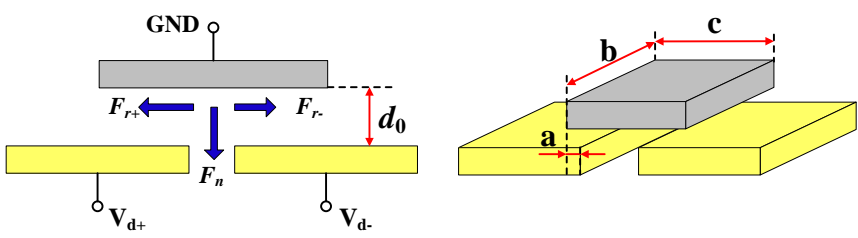

Fig. 6. Comb tooth on silicon (in gray) and electrodes on glass (in yellow)

The driving voltage is composed of DC and AC voltage,

$$
\left\{\begin{array}{l}
V_{d+}=V_{d c}+V_{a c} \sin \omega_{d} t \\
V_{d-}=V_{d c}-V_{a c} \sin \omega_{d} t
\end{array}\right.
$$

Among them, $\omega_{d}$ is the resonance frequency of drive mode.

So, the magnitude of electrostatic forces is shown below:

$$
\left\{\begin{array}{l}
F_{r}=F_{r+}-F_{r-}=\frac{\varepsilon b V_{+}^{2}}{2 d}-\frac{\varepsilon b V_{-}^{2}}{2 d}=\frac{2 \varepsilon b V_{d c} V_{a c} \sin \omega_{d} t}{d} \\
F_{n}=\frac{\varepsilon b a V_{+}^{2}}{2 d^{2}}+\frac{\varepsilon b a V_{-}^{2}}{2 d^{2}}=\frac{\varepsilon b a\left(V_{d c}{ }^{2}+V_{a c}{ }^{2} \sin ^{2} \omega_{d} t\right)}{2 d^{2}} \\
\left\|F_{n}\right\| /\left\|F_{r}\right\|=\frac{a\left(V_{d c}{ }^{2}+V_{a c}{ }^{2}\right)}{4 d V_{d c} V_{a c}}
\end{array}\right.
$$

And the design values are shown in Table 1.
Table 1 Design values of the proposed structure

\begin{tabular}{|c|c|c|c|c|}
\hline Item & $V_{d c} /(\mathrm{V})$ & $V_{a c} /(\mathrm{V})$ & $a /(\mathrm{m})$ & $d /(\mathrm{m})$ \\
\hline Value & 30 & 5 & $30 \mathrm{e}-6$ & $5 \mathrm{e}-6$ \\
\hline
\end{tabular}

As can be seen from the Equation (3) and Table 1, the frequency of $F_{r}$ is $\omega_{d}$, while the frequency of $F_{n}$ is $2 \omega_{d}$. And we can obtain that the ratio of $F_{n}$ to $F_{r}$ is 9.25 . It's true that the $F_{n}$ is larger than $F_{r}$. However, when the structure is driven in resonant frequency $\left(\omega_{d}\right)$, its horizontal displacement caused by $F_{r}$ will be magnified by $Q(Q=14296)$ times due to the resonance effect. But the vertical displacement will not, that's why the gyroscope can be driven by horizontal electrostatic force and the vertical electrostatic force can't generate obvious vertical displacement.

Suppose the number of comb teeth is $n$, so the horizontal electrostatic driving force for the proposed vibration structure is:

$$
F_{r, d r i v e}=n\left(F_{r+}-F_{r-}\right)=\frac{2 n \varepsilon b}{d} V_{d c} V_{a c} \sin \omega_{d} t
$$

As can be seen from the equation (4), the magnitude of the horizontal electrostatic driving force is related to the length of the comb teeth $(b)$ and the number of comb tooth $(n)$. In order to obtain as much driving force as possible, increasing the comb length $(b)$ is one way to enhance the driving force. But it will reduce the area of detection electrode which would decrease the detection sensitivity.

Reducing the width of single comb tooth $(c)$ to increase the number of comb teeth $(n)$ is another way to enhance the driving force, but the maximum of horizontal driving amplitude will be limited to the misalignment between comb teeth and electrodes, which could make the gyroscope work abnormally.

Therefore, in the design process of the proposed vibration structure, the influence of various factors on the sensitivity of gyroscope should be considered comprehensively.

\subsection{Design of the length of driving comb teeth}

On the condition that unit angular velocity is input, the displacement of vibrating mass in sensing direction $\left(S_{z}\right)$ can be described as:

$$
S_{z}=\frac{4 \varepsilon n Q_{d} V_{d c} V_{a c} \sin (\omega t)}{K_{s} \omega_{d} d_{0} \sqrt{\left(1-\left(\frac{\omega_{d}}{\omega_{d}+\Delta \omega}\right)^{2}\right)^{2}+\left(\frac{\omega_{d}}{\left(\omega_{s}+\Delta \omega\right) Q_{s}}\right)^{2}}} * b
$$

When the changes in the capacitance gap $(\Delta d)$ is equal to the displacement of vibration mass in sensing direction $\left(S_{z}\right)$, the variation of sensing capacitance $\left(\Delta C_{s}\right)$ could be described as:

$$
\Delta C_{s}=\frac{4 \varepsilon^{2} n w_{m} Q_{d} V_{d c} V_{a c} \sin (\omega t)}{K_{s} d_{0}^{3} \omega_{d} \sqrt{\left(1-\left(\frac{\omega_{d}}{\omega_{d}+\Delta \omega}\right)^{2}\right)^{2}+\left(\frac{\omega_{d}}{\left(\omega_{s}+\Delta \omega\right) Q_{s}}\right)^{2}}} *\left(l_{m}-b\right) b
$$

Among them, $w_{m}$ is the width of mass and $l_{m}$ is the length of mass. $d_{0}$ is the initial gap between silicon structure and glass substrate. As can be seen from the equation (6), the variation of 
sensing capacitance varies with the length of comb $(b)$. And when the length of comb is half of the mass, the variation of sensing capacitance reaches the maximum.

$$
b=\frac{l_{m}}{2}
$$

\subsection{Design of the width of driving comb teeth}

The driving amplitude is determined by the width of driving comb tooth in some degree. As can be seen in Fig. 7, there are two dimensional parallel plate capacitances with part of area overlapped.

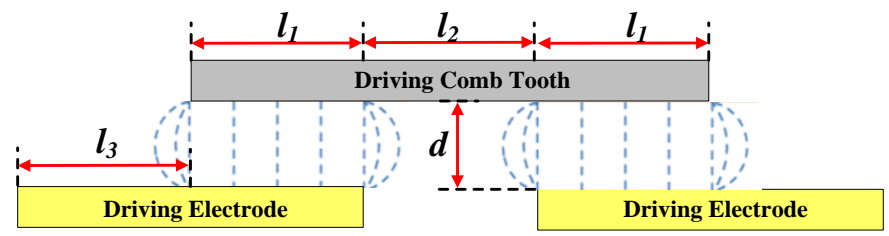

Fig. 7. Parallel plate capacitances with part of area overlapped

When the edge effect is not considered, the capacitor can be described as:

$$
C_{0}=\frac{\varepsilon l_{1}}{d}
$$

When the edge effect is taken into consideration, the capacitor can be described as:

$$
C_{f}=\frac{\varepsilon l_{1}}{d}+\frac{\varepsilon}{\pi}\left\{1+\ln \left[1+\frac{2 \pi l_{1}}{d}+\ln \left(1+\frac{2 \pi l_{1}}{d}\right)\right]\right\}
$$

As can be seen from the equation (9), if the edge effect is taken into consideration, the capacitor would not have the linear relationship with the overlap length $\left(l_{1}\right)$ any more, and size of the capacitor would become bigger.

The relative error between $C_{0}$ and $C_{f}$ is defined as $\delta c$, so the value of $\delta c$ could reflect the extent of the edge effect.

$$
\delta c=\frac{\left|C_{f}-C_{0}\right|}{C_{0}}
$$

The relative error between $C_{0}$ and $C_{f}$ is calculated and plotted with the increasing of the ratio of overlap length to gap, as shown in Fig. 8 (a). From the calculation result curve, we can see that when the ratio $\left(l_{1} / d\right)$ is bigger than 10 , the relative error decreases and becomes almost unchanged.

The length of nonoverlapping area $\left(l_{2}\right)$ is another factor which has effect on the extent of edge effect. So, in order to make clear how the length of nonoverlapping area affects the capacitor, simulation is carried out between the capacitor and the ratio of nonoverlapping length to gap $\left(l_{2} / d\right)$. As can be seen in Fig. 8 (b), when the ratio $\left(l_{2} / d\right)$ is smaller than 10 , the nonlinear effect is obvious. When the ratio is bigger than 10 , the capacitor is almost linear with the ratio without nonlinear effect.

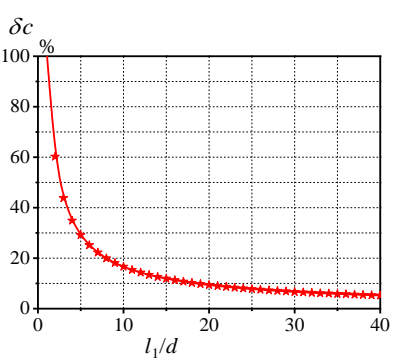

(a)

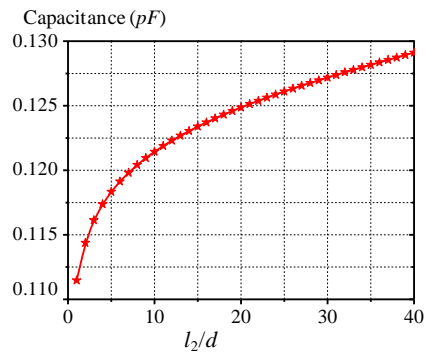

(b)
Fig. 8. (a) The relative error varies with the ratio of $l_{1} / d$. (b) The capacitor varied with the ratio of $l_{2} / d$

Taking all the analysis before into consideration, the width of driving comb teeth should be at least as 30 times as the capacitance gap to avoid the nonlinear effect of the edge effect. The optimized structural parameters of proposed vibration structure are determined by theoretical analysis, finite element simulation and experimental verification. The modal simulation was carried out in COMSOL, and simulation results of the structure are shown in Fig. 9.

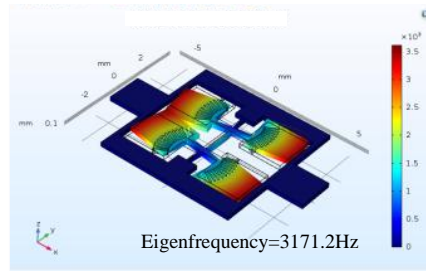

(a) The $2^{\text {nd }}$ order mode $(3171.2 \mathrm{~Hz})$

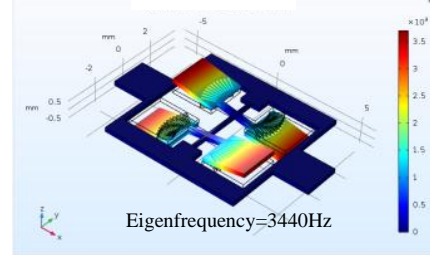

(b) The 3rd order mode $(3440 \mathrm{~Hz})$
Fig. 9. The modal simulation results

The drive mode shown in Fig. 9 (a) is the flexural vibration of the rectangle beam. And the sense mode shown in Fig. 9 (b) is the torsional vibration of the rectangle beam.

\section{DYNAMIC CHARACTERISTIC ANALYSIS}

The sensitivity and thermal noise are two main factors of the dynamic characteristic of gyroscope. So, improving the sensitivity and decreasing the thermal noise are important for the gyroscope to improve the performances.

\subsection{Analysis of capacitance sensitivity}

According to the proposed structure of the design, the theoretical calculation of sensitivity was carried out. The calculation of the mechanical sensitivity $(\mathrm{m} / \% / \mathrm{s})$ of the detection displacement is shown in equation (11):

$$
S=\frac{y_{s}}{\Omega_{z}}=\frac{2 \omega_{d} m x_{0} / k_{s s}}{\sqrt{\left[1-\left(\frac{\omega_{d}}{\omega_{s}}\right)^{2}\right]^{2}+\left(\frac{\omega_{d}}{\omega_{s} Q_{s}}\right)^{2}}}
$$

Among them, $\omega_{d}$ is the frequency of drive mode. $\omega_{s}$ is the frequency of sense mode. $x_{0}$ is the horizontal vibration amplitude 
of drive mode. $Q_{s}$ is the $Q$-factor of sense mode. $k_{s s}$ is the stiffness coefficient of sense axis.

As can be seen from the equation (11), the displacement sensitivity in sense direction of the gyroscope is proportional to the magnitude of the horizontal vibration amplitude $x_{0}$. Therefore, the displacement sensitivity in sense direction can be increased by increasing $x_{0}$.

The displacement sensitivity in sense direction is the displacement shift in sense direction when unit angular speed is input. Sensitivity of sense capacitance $\left(\mathrm{pF}^{\circ} / \mathrm{s}\right)$ can be obtained using calculation equation of the capacitance shift:

$$
\Delta C=\frac{\varepsilon A^{\prime}}{d^{\prime}}-\frac{\varepsilon A_{0}}{d_{0}} \approx \varepsilon A_{0} \frac{S}{d_{0}\left(d_{0}-S\right)}
$$

As can be seen from the equation (12), the sensitivity of the sense capacitance is mainly related to the displacement sensitivity in sense direction and the initial capacitance gap. Compared to the conventional one, the proposed structure could have greater horizontal displacement $x_{0}$, and the initial capacitive gap $d_{0}$ could be reduced which would further enhance the sensitivity of the gyroscope.

\subsection{Analysis of thermal mechanical noise}

Thermal mechanical noise is caused by molecular agitation inside springs, beams and other mechanical parts, and it is the equivalent of Johnson's noise in resistors, which represents a major noise source and can be a practical limit in stability for MEMS vibrating gyroscopes [28].

The thermal mechanical noise equivalent angular velocity of a MEMS vibrating gyroscope can be described as:

$$
\Omega_{T M N}=\frac{1}{x_{0}} \sqrt{\frac{k_{b} T \omega_{s} B}{m \omega_{d}^{2} Q_{s}}}
$$

Where $k_{b}$ is the Boltzman constant, $T$ is the absolute temperature in degrees Kelvin, $\omega_{d}$ and $\omega_{s}$ are the resonant frequency of drive and sense mode, $B$ is the bandwidth of the gyroscope, $m$ is the vibrating mass, $Q_{s}$ is the quality factor of sense mode and $x_{0}$ is the dynamic displacement along driving direction.

According to equation (13), when the structure of a MEMS gyroscope is confirmed, an effective way to decrease thermal mechanical noise equivalent angular velocity is to increase amplitude of drive mode $\left(x_{0}\right)$. So, compared with vertical electrostatic force excited gyroscope, stability limitation of the proposed structure is much smaller, indicating that the horizontal electrostatic force excited 'Butterfly' gyroscope has more potential in high precision applications.

\section{PROCESSING TECHNOLOGY AND TEST RESUlTS}

\subsection{Processing technology}

The prototypes of the gyroscope are fabricated on a single crystal silicon wafer. The main fabrication process is illustrated in Fig. 10.

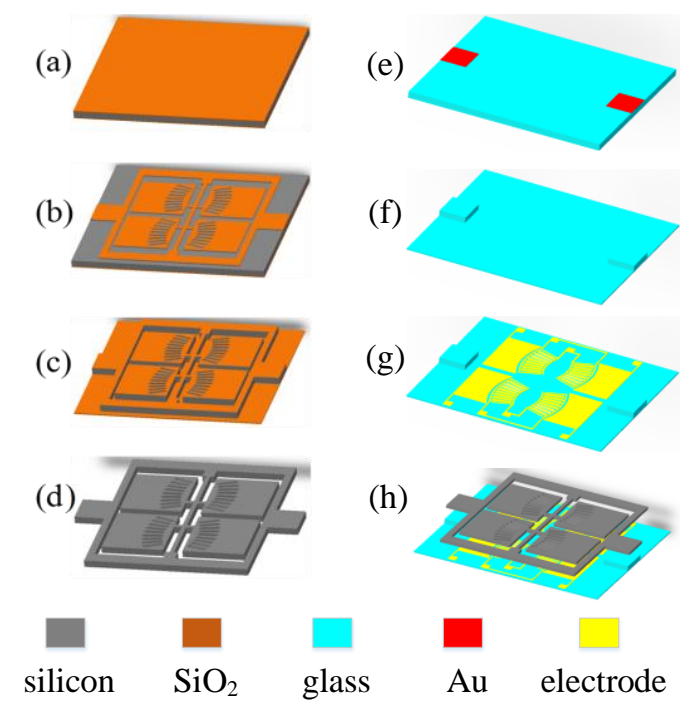

Fig. 10. The processing technology of the proposed butterfly gyroscope

The silicon process starts with a double-side polished, $240 \mu \mathrm{m}$ thick, (100) oriented n-type silicon wafer. Firstly, oxidation layer $\left(\mathrm{SiO}_{2}\right)$ is formed on both sides of the silicon wafer, as shown in Fig. 10 (a), which will serve as the mask mater material in the following DRIE process. Then single side lithography in performed with the appointed mask on one side of $\mathrm{SiO}_{2}$ to define the mechanical structure, as shown in Fig. 10 (b). Then the wafer is etched by DIRE process until the etching reaches through the wafer to form the designed structure, as shown in Fig. 10 (c). Finally, the silicon structure is realized by removing the $\mathrm{SiO}_{2}$ layer, as shown in Fig. 10 (d).

Meanwhile, the glass substrate process starts with evaporating gold on the surface of the glass to form the masking layer for glass etching with buffered oxide etchant. The $\mathrm{Cr} / \mathrm{Au}$ film is patterned by lithography and wet etching, as shown in Fig. 10 (e). Then the glass is etched in buffered oxide etchant to form cavities in the glass substrate, as shown in Fig. 10 (f). After removing the $\mathrm{Au}$ film, the aluminum electrodes are sputtered and patterned in the cavities by lithography and wet etching, as shown in Fig. 10 (g). Afterward, the silicon wafer and the glass substrate with patterned electrodes are bonded together, as shown in Fig. 10 (h).

The SEM view of the silicon structure sidewall after DRIE processing is present in Fig. 11, accordingly, steepness and roughness error of sidewall are less than 1 degree and $200 \mathrm{~nm}$, respectively.

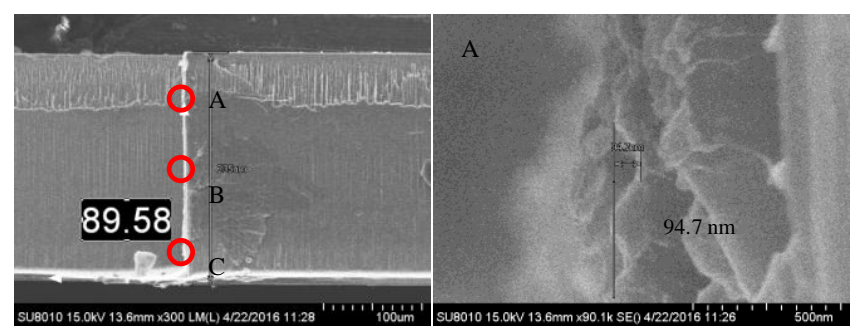

$\begin{array}{lll}\text { (a) Steepness error of the sidewall } & \text { (b) Roughness of point A }\end{array}$ 


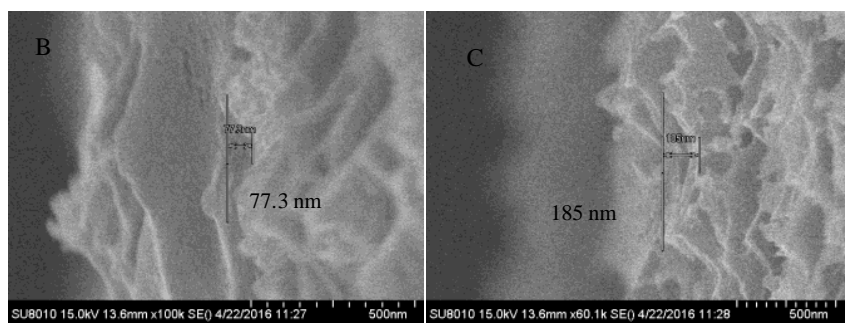

(c) Roughness of point $\mathrm{B}$

(d) Roughness of point C

Fig. 11. SEM view of silicon structure sidewall after DRIE process

And then, the proposed gyroscope was packaged in ceramic tubes, as shown in Fig. 12.

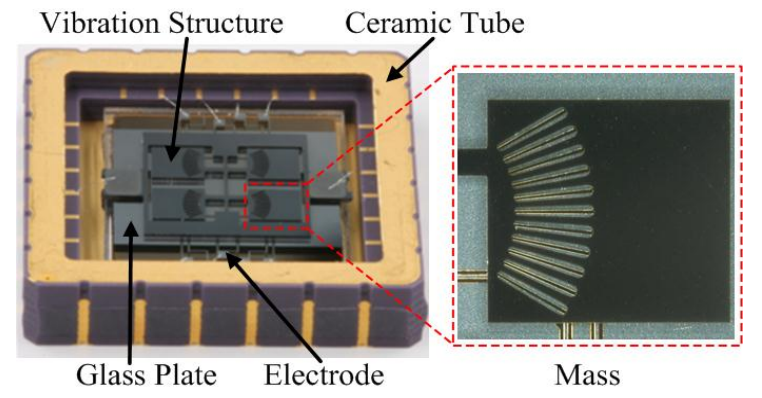

Fig. 12. The proposed butterfly gyroscope packaged in a ceramic tube

\subsection{Experiments and results}

The performance test of gyroscopes in ceramic tubes mainly included modal testing and zero bias stability testing.

FRA (Frequency Analysis Response) was used to conduct modal testing of the proposed gyroscope, and the results are shown in Fig. 13.
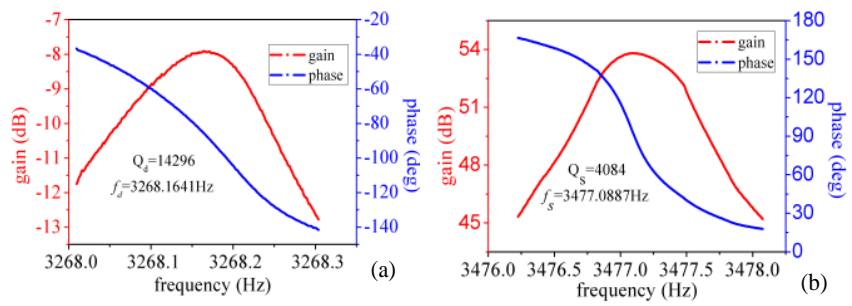

Fig. 13. Modal testing of the propposed gyroscope

The frequency response in driving direction is shown in Fig. 13 (a), the resonance frequency is $3268.16 \mathrm{~Hz}$ and the quality factor is 14296 . The frequency response in sensing direction is shown in Fig. 13 (b), the resonance frequency is $3477.09 \mathrm{~Hz}$ and the quality factor is 4084 .

The readout circuitry of the gyroscope consists of the drive and sense loop, and the schematic diagram of weak capacitance detection is shown in Fig. 14.

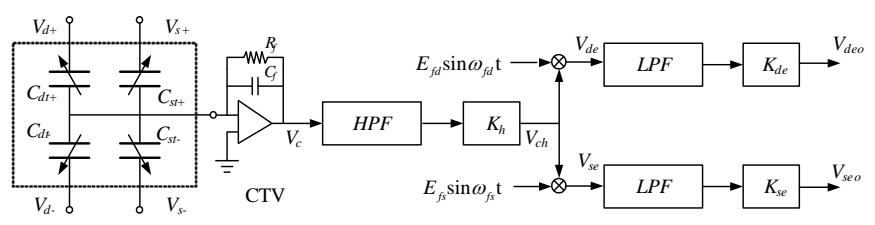

Fig. 14. The schematic diagram of weak capacitance detection
As can be seen in Fig. 14, the gyroscope can be equivalent to a five port device, and its equivalent circuit model is two pairs of differential capacitance with one common capacitance plate which is the silicon structure of gyroscope. $C_{d t+}$ and $C_{d t-}$ are the differential capacitance for driving axis, while $C_{s t+}$ and $C_{s t \text { - }}$ are the differential capacitance for sensing axis. The voltages applied on the differential capacitances are shown in equation (14):

$$
\left\{\begin{array}{l}
V_{d+}=V_{d c}+V_{a c} \sin \omega_{d} t+E_{f d} \sin \omega_{f d} t \\
V_{d-}=V_{d c}-V_{a c} \sin \omega_{d} t-E_{f d} \sin \omega_{f d} t \\
V_{s+}=E_{f s} \sin \omega_{f s} t \\
V_{s-}=-E_{f s} \sin \omega_{f s} t
\end{array}\right.
$$

Where, $V_{d c}$ is the DC bias, $V_{a c} \sin \omega_{d} t$ is the AC drive voltage, $E_{f d} \sin \omega_{f d} t$ is the high frequency carrier in driving axis, and $E_{f s} \sin \omega_{f s} t$ is the high frequency carrier in sensing axis. So, the end output voltages are shown in equation (15):

$$
\left\{\begin{array}{l}
V_{\text {deo }}=-\frac{K_{h} K_{d e} E_{f d}^{2} \Delta C_{d}}{2 C_{f}} \\
V_{\text {seo }}=-\frac{K_{h} K_{s e} E_{f s}^{2} \Delta C_{s}}{2 C_{f}}
\end{array}\right.
$$

From the equation (15), we can see that output voltage $V_{d e o}$ and $V_{\text {seo }}$ are proportional to the variation of the driving and sensing capacitance respectively. Thus, the variation of the driving and sensing capacitance can be effectively extracted and separated.

After that, the demodulated $V_{d e o}$ can be used for phase control and amplitude control to ensure a stabilized driving vibration amplitude as shown in Fig. 15.

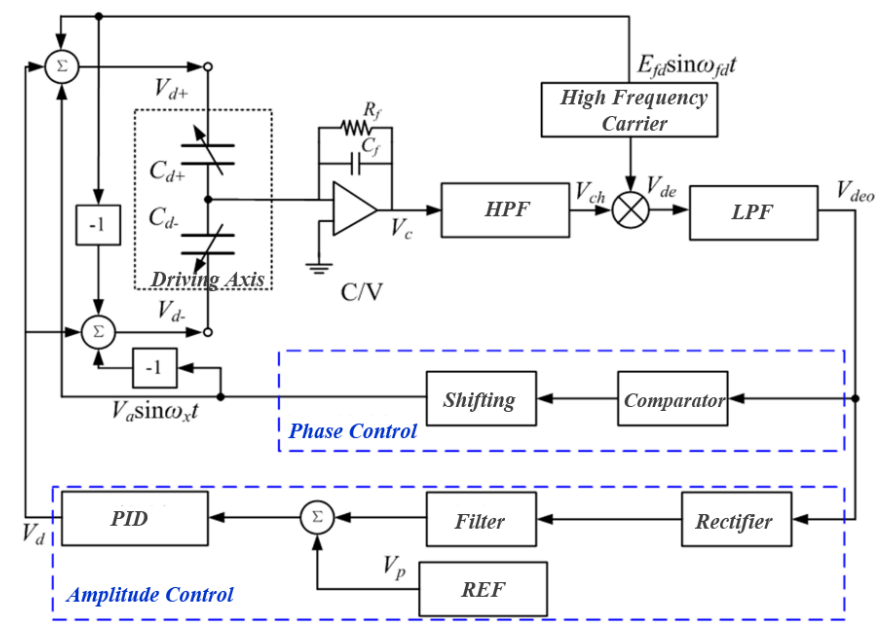

Fig. 15. The schematic diagram of driving closed loop circuit

Meanwhile, the variation of different sensing capacitors is transformed to a voltage signal modulated by carrier signal and driving signal. Then in the sense loop, after carrier demodulation and angular rate demodulation, a direct current voltage which is proportional to the angular rate will be extracted.

As can be seen from Fig. 16, the scale factor of the conventional butterfly micro-machined gyroscope was 0.508 $(\mathrm{mV} / \%)$. The scale factor of the proposed structure was 9.8 $(\mathrm{mV} / \%)$. 


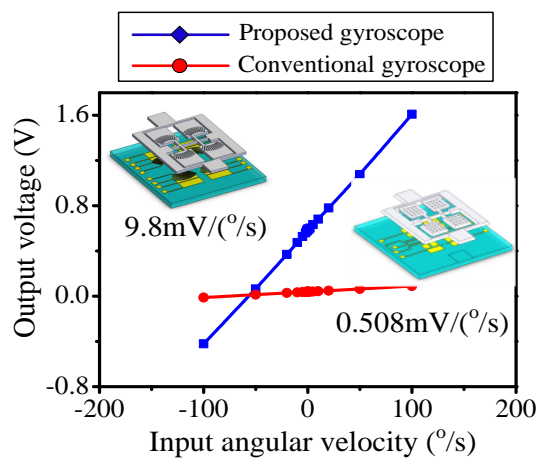

Fig. 16. The scale factor test of conventional and proposed gyroscopes

After the scale factor test, we can obtain the capacitance sensitivity and the driving amplitude, and the calculation results showed that the proposed gyroscope could generate much more driving amplitude than the conventional one, as shown in table 2.

Table 2. Comparison of the driving amplitudes

\begin{tabular}{|c|c|c|c|}
\hline & $\begin{array}{c}\text { Scale } \\
\text { factor } \\
/ \mathrm{mV} /(\% / \mathrm{s})\end{array}$ & $\begin{array}{c}\text { Capacitance } \\
\text { sensitivity } \\
/ \mathrm{F} /(\mathbf{o})\end{array}$ & $\begin{array}{c}\text { Driving } \\
\text { Amplitude } \\
/ \mathrm{m}\end{array}$ \\
\hline $\begin{array}{c}\text { Conventional } \\
\text { Gyroscope }\end{array}$ & 0.508 & $6.26 \mathrm{e}-17$ & $5.81 \mathrm{e}-6$ \\
\hline $\begin{array}{c}\text { Proposed } \\
\text { Gyroscope }\end{array}$ & 9.8 & $8.08 \mathrm{e}-16$ & $29.5 \mathrm{e}-6$ \\
\hline
\end{tabular}

Recently, the performance testing for the proposed gyroscope has been carried out, and it's testing results are shown in Fig. 17. The ARW of proposed gyroscope is measured to be $1.244 \mathrm{deg} / \sqrt{\mathrm{h}}$, and the bias stability reached $3.58 \mathrm{deg} / \mathrm{h}$.

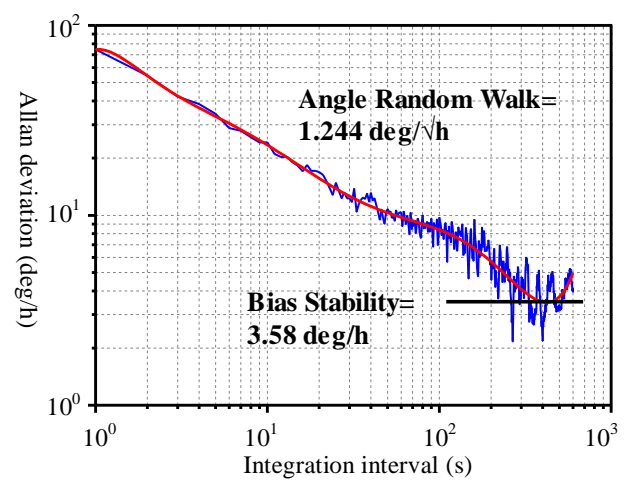

Fig. 17. The ARW and Bias Stability measurement results of the proposed gyroscope

Comparing to the advanced level of the tuning fork gyroscope performance, the proposed gyroscope in this paper has its own advantages as shown in table 3.

Table 3. Advanced High performance MEMS gyroscopes

\begin{tabular}{|c|c|c|c|c|c|c|c|c|}
\hline \multirow{2}{*}{\multicolumn{2}{|c|}{$\begin{array}{l}\text { Research } \\
\text { Institution }\end{array}$}} & \multirow{2}{*}{$\begin{array}{l}\mathrm{Q}_{\mathrm{s}} \\
\text { factor }\end{array}$} & $\begin{array}{l}\text { Split } \\
\text { Freq. }\end{array}$ & $\begin{array}{l}\text { Band } \\
\text { width }\end{array}$ & Range & ARW & $\begin{array}{c}\text { Bias } \\
\text { stability }\end{array}$ & \multirow{2}{*}{$\begin{array}{l}\text { Year } \\
\& \\
\text { Ref }\end{array}$} \\
\hline & & & $\mathrm{Hz}$ & $\mathrm{Hz}$ & $\% / \mathrm{s}$ & $\% / \sqrt{h}$ & $\% / \mathrm{h}$ & \\
\hline \multicolumn{2}{|c|}{ A*STAR } & N/A & 250 & 120 & \pm 500 & 0.45 & 9.6 & $\begin{array}{l}2017 \\
{[29]}\end{array}$ \\
\hline \multirow{3}{*}{ FE } & type1 & $60 \mathrm{k}$ & N/A & N/A & N/A & 0.17 & 4.4 & \multirow{3}{*}{$\begin{array}{l}2015 \\
{[30]}\end{array}$} \\
\hline & type2 & $60 \mathrm{k}$ & N/A & N/A & N/A & 0.1 & 1.1 & \\
\hline & type3 & $60 \mathrm{k}$ & N/A & N/A & N/A & 0.06 & 5.5 & \\
\hline \multicolumn{2}{|c|}{ SEU } & N/A & -90.2 & 47 & \pm 100 & 0.14 & 0.2 & $\begin{array}{l}2015 \\
{[31]}\end{array}$ \\
\hline \multicolumn{2}{|c|}{ This Paper } & 4084 & 200 & 100 & \pm 100 & 1.244 & 3.25 & 2018 \\
\hline
\end{tabular}

A*STAR, Agency for Science, Technology and Research, Singapore; FE, Fraunhofer ENAS, Germany; SEU, Southeast University, China.

From increasing the driving amplitude to improving the performance, it's a long way to go. There are much more efforts need to be made to improve the performance in the next step such as the processing accuracy, the readout circuit and so on.

\section{CONCLUSION}

The horizontal driving amplitude of traditional butterfly gyroscope with a slanted vibration beam is subjected to the vertical driving displacement and initial capacitance gap, which, to a great extent, limits its sensitivity. So, in this paper, a new type of vibration structure with rectangle vibration beam was proposed. Through driving the proposed structure by horizontal electrostatic force instead of vertical electrostatic force, signalnoise ratio could be improved by increasing dynamic amplitude of drive mode and decreasing initial gap between silicon and glass, simultaneity. The key dimension of comb tooth was designed, and the fabrication processing was presented. And then performance test of the gyroscopes in ceramic tubes was conducted. The scale factor test and the calculation results showed a great enhancement in the driving amplitude. And the ARW of proposed gyroscope was measured to be $1.244 \mathrm{deg} / \sqrt{\mathrm{h}}$, and the bias stability reached $3.58 \mathrm{deg} / \mathrm{h}$.

Indeed, the performance of the reported gyroscope is not impressive enough. However, the mechanical thermal noise of the gyroscope obtained by the theoretical analysis and calculation is $0.23^{\circ} / \mathrm{h} / \sqrt{ } \mathrm{Hz}$ showing a good precision potential. And it has achieved considerable results in mechanical sensitivity improvement in this essay.

Now, we are working on improving the performance of gyroscope from the following aspects. (1) As for the structural optimization, the processing technology could be further optimized to reduce the fabrication errors and improve the fabrication quality. (2) What's more, the silicon electrode should be applied instead of glass electrode to decrease the structural stress which is brought by the different thermal expansion coefficients between the silicon and the glass. (3) In terms of the signal processing circuit, it could be improved by adding error control and error compensation parts which could cut down the noise and improve the performance. We believe that the reported gyroscope will achieve good performance after these efforts.

\section{ACKNOWLEDGMENT}

This work was supported by the National Natural Science Foundation of China (NSFC, Grant No. 51505490).

\section{REFERENCES}

[1] Paddy French, Gijs Krijnen and Fred Roozeboom. "Precision in harsh environments," Microsystems \& Nanoengineering, 2, 1-12, 2016.

[2] D. Yang, J.-K. Woo, S. Lee, J. Mitchell, A. D. Challoner, and K. Najafi, "A Micro Oven-Control System for Inertial Sensors," Journal Of Microelectromechanical Systems, vol. 26, pp. 507-518, Jun 2017.

[3] J. Sung, J. Y. Kim, S. Seok, H. J. Kwon, M. Kim, G. Kim, et al., "A gyroscope fabrication method for high sensitivity and robustness to fabrication tolerances," Journal Of Micromechanics And Microengineering, vol. 24, Jul 2014. 
[4] N. Minh Ngoc, H. Nhat Sinh, N. Long Quang, C. Hoang Manh, and V. Hung Ngoc, "Z-Axis Micro-machined Tuning Fork Gyroscope with Low Air Damping," Micromachines, vol. 8, Feb 2017.

[5] C. Yang and H. Li, "Digital Control System for the MEMS Tuning Fork Gyroscope Based on Synchronous Integral Demodulator," Ieee Sensors Journal, vol. 15, pp. 5755-5764, Oct 2015.

[6] Y. Guan, S. Gao, L. Jin, and L. Cao, "Design and vibration sensitivity of a MEMS tuning fork gyroscope with anchored coupling mechanism," Microsystem Technologies, vol. 22, pp. 247-254, 2015.

[7] S. A. Zotov, B. R. Simon, I. P. Prikhodko, A. A. Trusov, and A. M. Shkel, "Quality Factor Maximization Through Dynamic Balancing of Tuning Fork Resonator," Ieee Sensors Journal, vol. 14, Aug 2014.

[8] I. P. Prikhodko, S. A. Zotov, A. A. Trusov, and A. M. Shkel, "Foucault pendulum on a chip: Rate integrating silicon MEMS gyroscope," Sensors And Actuators a-Physical, vol. 177, pp. 67-78, Apr 2012.

[9] E. Tatar, T. Mukherjee, and G. K. Fedder, "Stress Effects and Compensation of Bias Drift in a MEMS Vibratory-Rate Gyroscope," Journal Of Microelectromechanical Systems, vol. 26, pp. 569-579, Jun 2017.

[10] B. S. Park, K. Han, S. Lee, and M. Yu, "Analysis of compensation for a g-sensitivity scale-factor error for a MEMS vibratory gyroscope," Journal of Micromechanics and Microengineering, vol. 25, p. 115006, 2015.

[11] S. Sonmezoglu, S. E. Alper, and T. Akin, "An Automatically ModeMatched MEMS Gyroscope With Wide and Tunable Bandwidth," Journal of Microelectromechanical Systems, vol. 23, pp. 284-297, Apr 2014.

[12] M. Putty and K. Najafi, "A Micromachined Vibrating Ring Gyroscope," Tech. Digest of the 1994 Solid State Sensors and Actuators workshop, Hilton Head Island, SC, pp. 213-220.

[13] Ayazi, Farrokh ; Najafi, Khalil. "A HARPSS polysilicon vibrating ring gyroscope," Journal of Microelectromechanical Systems, 2001 , Vol. 10, No. 2, pp. 169-179.

[14] Johari H,Ayazi F. "High-frequency capacitive disk gyroscopes in (100) and (111) silicon," Proc. of 20th IEEE Inter. Conf. on Micro Electro Mechanical Systems, 2007: 47-50.

[15] X. Zhou, D. Xiao, X. Wu, Y. Wu, Z. Hou, K. He, et al., "Stiffness-mass decoupled silicon disk resonator for high resolution gyroscopic application with long decay time constant (8.695 s)," Applied Physics Letters, vol. 109, Dec 262016.

[16] SHAO P, TAVASSOLI V, MAYBERRY C L, et al. "A 3D-HARPSS polysilicon micro-hemispherical shell resonating gyroscope: Design, fabrication, and characterization," IEEE Sensors Journal, 2015, 15(9): 4974-4985.

[17] Cho JY, Woo JK, Yan JL, Peterson RL, Najafi K (2014) Fused-silica micro birdbath resonator gyroscope (mu-BRG). J Microelectromech Syst 23:66-77.

[18] D. Senkal, M. J. Ahamed, A. A. Trusov, and A. M. Shkel, "Achieving Sub-Hz Frequency Symmetry in Micro-Glassblown Wineglass
Resonators," Journal of Microelectromechanical Systems, vol. 23, pp. 3038, Feb 2014.

[19] G. Andersson, N. Hedenstierna, P. Svensson, and H. Pettersson, "A novel silicon bulk gyroscope," in Proceedings of International Conference on Transducers, 1999, pp. 902-905.

[20] N. Hedenstierna, S. Habibi, S. M. Nilsen, T. Kvisteroy, and G. U. Jensen, "Bulk micro-machined angular rate sensor based on the'butterfly'-gyro structure," in Micro Electro Mechanical Systems, 2001. MEMS 2001. The 14th IEEE International Conference on, 2001, pp. 178-181.

[21] D. Xiao, S. Cao, Z. Hou, Z. Chen, X. Wang, and X. Wu, "Enhanced sensitivity in a butterfly gyroscope with a hexagonal oblique beam," Aip Advances, vol. 5, Apr 2015.

[22] J. Su, D. Xiao, X. Wu, Z. Hou, and Z. Chen, "Improvement of bias stability for a micro-machined gyroscope based on dynamic electrical balancing of coupling stiffness," J. Micro/Nanolith. MEMS MOEMS 2013.

[23] Z. Hou, Z. Chen, X. Wu, J. Su, and D. Xiao, "Transition interface for improving the temperature characteristics of micro-machined gyroscopes," Microsyst Technol 2014.

[24] Y. Dong, M. Kraft, N. Hedenstierna, and W. Redman-White, "Microgyroscope control system using a high-order band-pass continuous-time sigma-delta modulator," Sensors and Actuators A: Physical, 2008.

[25] J. B. Su, D. B. Xiao, X. Z. Wu, Z. Q. Hou, and Z. H. Chen, "Improvement of bias stability for a micro-machined gyroscope based on dynamic electrical balancing of coupling stiffness," Journal Of MicroNanolithography Mems And Moems, vol. 12, Jul-Sep 2013.

[26] D. Xiao, J. Su, Z. Chen, Z. Hou, X. Wang, and X. Wu, "Improvement of mechanical performance for vibratory microgyroscope based on sense mode closed-loop control," Journal of Micro/Nanolithography, MEMS, and MOEMS, vol. 12, pp. 023001-023001, 2013.

[27] D. Xiao, X. Wu, X. Wang, Z. Chen, Z. Zhou, and Z. Hou, "A novel fabrication method based on an after thermal oxidation process for the realization of silicon-beams with ormative polygon cross sections shapes," Microsyst Techno, 2013.

[28] V. Annovazzi-Lodi and S. Merlo, "Mechanical-thermal noise in micromachined gyros," Microelectronics Journal, vol. 30, pp. 1227-1230, 1999.

[29] G.Q. Wu, G.L. Chua, Y.D. Gu, "A dual-mass fully decoupled MEMS gyroscope with wide bandwidth and high linearity," Sensors and Actuators A: Physical, pp. 50-56, 2017.

[30] Roman Forke, Karla Hiller, Susann Hahn, Stefan Konietzka, Tim Motl, Daniel Kohler, Steffen Heinz, Detlef Billep, Thomas Gessner, "Yet Another Tuning Fork Gyroscope," IEEE International Symposium on the Inertial Sensors and Systems, pp. 1-4, 2015.

[31] Cheng Yang and Hongsheng Li, "Digital Control System for the MEMS Tuning Fork Gyroscope Based on Synchronous Integral Demodulator," IEEE Sensors Journal, VOL. 15, pp. 5755-5764, 2015. 